Marie (Aurélie) Thériault Université de Montréal

\section{Apprendre et enseigner à la formation générale des adultes: une recherche ethnopédagogique auprès de finissants et finissantes ainsi que d'enseignantes}

Learning and teaching at Adult Education: sanr ethnopedagogical research with close graduate and teachers

doi:10.18162/fp.2016.381

\title{
ésumé
}

La formation générale des adultes est sollicitée par des apprenants présentant des profils particuliers.

Documenter le processus de réussite des individus inscrits en $4 e$ et 5 e années du secondaire ayant vécu un parcours scolaire hors norme permet de comprendre les pratiques garantissant persévérance et insertion sociale. Nous appuyant sur les modèles andragogiques, nous proposons une méthodologie destinée autant aux apprenants de l'éducation des adultes qu'à leurs pédagogues. Nos résultats font état des récits de vie des finissants et de données relatives à leur perception des pratiques pédagogiques et à la modification de la vision de leur insertion sociale. Pour les intervenantes, la recherche documente la trajectoire de leur exercice professionnel et leur pédagogie.

Mots-clés

éducation, adultes, formation, histoire de vie, ethnographie, trajectoire, réussite scolaire

Abstract

General adult education is sought by learners with specific profiles. By documenting the process of success

of individuals enrolled in secondary's 4th and 5th grades, who have followed out of the ordinary school paths, we are now able to understand practices that warrant perseverance and social insertion. By relying on the concept of andragogy, we suggest a methodology dedicated to both practitioners and learners. Our results show life stories of graduates and data on their perceptions of teaching practices and changes in vision of their social inclusion. For practitioners, this research documents the trajectory of their professional practice and their pedagogy.

\section{Introduction}

La persévérance et la réussite scolaires constituent un défi, particulièrement à l'éducation des adultes où on retrouve une diversité de profils et de parcours chez les apprenants. La Commission d'étude sur la formation professionnelle et la formation socioculturelle des adultes (CÉFA) entreprend, dans les années 80 , avec en arrière-fond la prise en charge de ce défi, des travaux d'envergure dont l'objet se fond, dans la lignée du Rapport Parent, sur l'urgence de réorganiser les services d'éducation des adultes tout en conservant la spécificité propre de cet enseignement sur le double plan de la formation et de l'employabilité. La notion de formation de base date de cette décennie : on fait le diagnostic qu'elle est lacunaire chez plusieurs adultes, cela nuisant au plein développement de leur insertion sociale (Inchauspé, 1999). La CÉFA plaide alors pour un système public qui sache inviter davantage d'adultes à finir leurs études secondaires ou à entreprendre des études collégiales. En 1994, un régime pédagogique propre aux adultes et à la formation professionnelle est adopté. Suivent les États généraux sur l'éducation et ses chantiers (1995-1996) qui permettent de faire le point sur le système d'éducation et de lancer les prolégomènes d'une vaste réforme basée sur la notion de compétences. La Politique de l'éducation des adultes et de la formation continue adoptée en 2002 se situe dans la lignée de cette réflexion. Le Régime pédagogique de la formation générale des adultes (FGA), prévu par la Loi sur l'instruction publique, est modifié en 2007-2008 pour s'arrimer à la réforme et à la préparation à la formation collégiale. Dix services d'entrée en formation sont prévus pour s'adapter aux profils d'un nombre grandissant d'apprenants qui s'inscrivent dans des centres d'éducation des adultes (CÉA). La persévérance et la réussite scolaires de ces adultes sont un enjeu pour plusieurs raisons. D'abord, l'offre de 
formation évolue ainsi que les attentes des employeurs. Ensuite, le nombre d'adultes étudiant dans les CÉA croît, surtout chez les 16-24 ans, laissant apparaître, encore en 2015, un problème persistant de diplomation. Enfin, ces adultes font face à nombre de difficultés relatives à leur condition : parcours scolaires et de vie complexes, besoins de qualifications, précarité économique, celle des femmes en particulier. Des défis sont posés à la FGA où le fonctionnement pédagogique est particulier à ce système. La majorité des adultes fait appel à la formation de base commune $(\mathrm{FBC}), 1^{\mathrm{re}}$ et $2^{\mathrm{e}}$ années du secondaire, ou la formation de base diversifiée (FBD), $3^{\mathrm{e}}, 4^{\mathrm{e}}$ et $5^{\mathrm{e}}$ années du secondaire (MELS, 2009). Cependant, les adultes en fin de parcours ont formé notre objet d'études. Via l'établissement de leur portrait ethnopédagogique, il est possible de saisir le processus qui conduit à la réussite des études secondaires chez des individus ayant vécu un parcours scolaire hors norme ainsi que de comprendre les éléments issus des pratiques pédagogiques qui garantissent la persévérance scolaire et les conditions par lesquelles une insertion sociale devient possible. La notion de relation pédagogique sera donc étudiée sous l'angle de son impact sur la persévérance scolaire des adultes.

\section{Problématique}

La clientèle fréquentant l'éducation des adultes croît, si bien qu'en 2010-2011, 306574 adultes fréquentaient les centres par rapport à 247 459, en 2003-2004 (Ministère de l'Éducation, du Loisir et du Sport [MELS], 2009-2011). La majorité d'entre eux (2010-2011) étaient inscrits en formation de base diversifiée et en préparation aux études postsecondaires, formant ainsi la cohorte dominante de tous les effectifs de ce secteur d'enseignement. Pour l'année 2010-2011,68 295 personnes obtenaient le DES au secteur des jeunes alors que 14771 l'obtenaient au secteur des adultes, ce qui est considérable. Ce fait préoccupe grandement les directions et les enseignants œuvrant dans le secteur et renvoie au rapport de la CÉFA (1982. Pour comprendre cette question, il convient de placer l'adulte au centre de son projet de formation et de considérer les difficultés d'ordre psychologique, pédagogique et social qui l'ont conduit dans son parcours de formation, tout en considérant également les conditions par lesquelles il a su, via la structure de l'éducation des adultes, le redéployer et obtenir un diplôme lui permettant d'effectuer une transition d'insertion sociale. Il s'agit donc de s'ancrer dans un positionnement théorique qui s'appuie sur le modèle même de l'éducation des adultes et d'étudier simultanément ces dimensions, directement dans le milieu en incluant apprenants, intervenants et chercheure, ce qui n'a pas été fait explicitement jusqu'à maintenant. Les recherches existantes portent beaucoup sur les jeunes adultes faiblement scolarisés (Villemagne et Myre-Bisaillon, 2015) ainsi que sur le décrochage scolaire plutôt que sur le raccrochage scolaire (Marcotte, Villatte et Lévesque, 2014). Nous préférons les termes de prolongation scolaire, car la recherche a mis en évidence que les adultes fréquentant la FGA ont un profil de persévérance beaucoup plus qu'un profil de décrochage (Rousseau et al., 2010). L'intérêt de comprendre les parcours scolaires et de vie de ces adultes (Solar et Thériault, 2013) provient de cette observation. Les recherches se sont également intéressées à faire état des difficultés des adultes, sans toutefois étudier la question de la résolution de ces difficultés par l'obtention du DES, le diplôme essentiel pour l'insertion sociale d'un individu (Royer, 2014).

Le modèle andragogique joue un rôle central dans la persévérance de l'adulte (Doray et Bélanger, 2005) et a fortiori à la FGA. Il soutient l'adulte dans la création de projets de scolarisation et de vie, assurant ainsi son employabilité et son insertion sociale. L'enseignant joue un rôle important dans ce processus 
en s'adaptant, par exemple, au profil de l'apprenant. Potvin, Voyer et Bourdon (2014) démontrent que pédagogie et insertion sociale vont de pair avec persévérance et transition de vie en ce qu'une pédagogie centrée sur les besoins de l'apprenant le soutient dans la réussite de ses objectifs comme adulte. L'adaptation à l'apprenant est alors directement prise en charge par le milieu et fait partie des valeurs pédagogiques véhiculées par ce même milieu. Dans cet esprit, notre recherche insiste sur l'importance de bien comprendre les liens qui existent entre le parcours scolaire et de vie, le parcours pédagogique à la FGA, la persévérance dans l'objectif de l'obtention d'un diplôme qualifiant et l'insertion sociale. Doucet, Gauthier, Bizot et Maltais (2013) font d'ailleurs état de conditions éducationnelles appelées à régir l'inclusion scolaire à l'éducation des adultes et invitent, eux aussi, à la recherche collaborative avec le milieu. Cette recherche se situe donc dans un contexte où les actants du monde de l'éducation et de la recherche se sentent concernés par le phénomène de la prolongation des études secondaires et par l'insertion sociale des adultes moins scolarisés, ce qui passe évidemment par leur inclusion à court, à moyen et à long terme dans des programmes de formation et en emploi (Commission d'étude sur la formation des adultes, 1982; Gouvernement du Québec, 2002; Table des responsables de l'éducation des adultes et de la formation professionnelle des commissions scolaires, 2013). Conséquemment, cette recherche s'applique à répondre aux questions suivantes.

\section{Questions de recherche}

1. Quels sont les caractéristiques, les parcours scolaire et de vie (sur le plan psychologique, pédagogique et social) des apprenants de la FGA qui ont persévéré et obtenu leur DES?

2. Quelles sont les difficultés vécues et qu'est-ce qui explique, sur le plan pédagogique, leur réussite?

3. Quelle perception ont-ils de leur insertion sociale au terme de leur parcours à la FGA?

4. Qui sont les intervenants de la FGA et comment définissent-ils les concepts relatifs à la pédagogie et à l'insertion sociale des apprenants?

\section{Cadre conceptuel et théorique}

L'ethnographie permet d'approfondir les questions de cette recherche. Nous avons adapté son design au contexte de l'éducation des adultes à partir du modèle andragogique de Bélanger (2011) et de la théorie de l'autoformation de Carré et Moisan (2002). Le modèle de Bélanger est issu des acquis de la Sixième Conférence internationale sur l'éducation des adultes (CONFINTEA VI) tenue par l'UNESCO (2009). Ce modèle suppose que les adultes aient droit à une éducation de base tout au long d'une vie (Life long) qui s'adresse aux dimensions profondes de leur être (Life wide) et qui se colle sur l'expérience de l'apprenant (Life deep). La participation de l'adulte à des activités d'apprentissage repose sur ses aspirations et la réponse que la société lui fait en termes d'offre et de structure éducatives. Ce modèle permet de cadrer la problématique générale relevant de la diversité des parcours des adultes et de leur droit à une éducation de base pleine et entière en se positionnant par rapport à la formation générale des adultes, qui est vue ici à la fois comme une offre éducative et comme une structure éducative au sein de laquelle l'apprenant vit son expérience, et qui le conduit à exercer son libre arbitre dans la poursuite de son parcours de formation et d'insertion sociale. Pour ce qui concerne la théorie 
de l'autoformation, Carré et Moisan (2002), qui s'appuient sur les travaux de Long (1989), analysent la formation d'un adulte selon trois angles : un angle psychologique (objectifs scolaires et de vie); un angle pédagogique (en lien avec la perspective éducative); et un angle sociologique (l'angle du fait social). En effet, les parcours scolaires et de vie sont influencés par divers facteurs, notamment d'ordre psychologique (motivation, désir de réussite, de persévérer, estime de soi), pédagogique (relation avec l'enseignant, réalisation des objectifs, utilisation des ressources) et sociologique (offre éducative, fonctionnement du CÉA, adaptation, insertion sociale). En regard de la problématique psychologique, l'autoformation repose sur la capacité psychologique de l'apprenant de contrôler ses apprentissages en fonction de la perception qu'il développe de ses objectifs globaux, ici, son parcours à la FGA. De cette problématique découle notre préoccupation pour le portrait ethnopédagogique de l'adulte. En ce qui a trait à la problématique pédagogique, elle relève de la capacité qu'a l'apprenant de contrôler les composantes du projet d'apprentissage : lieux, durée, ressources, appui. De cette problématique découle notre intérêt pour la question de la pédagogie. Pour ce qui regarde la problématique sociologique, la formation est perçue comme un élément vivant de la société dont les adultes dépendent et dont ils sont maîtres du point de vue de leurs choix formels. De cette problématique découle notre intérêt pour les questions de la persévérance et de l'insertion sociale en lien avec la FGA. Ces trois angles ont donc été retenus pour la recherche. La transition de la FGA aux études collégiales ou dans le monde du travail est en soi une transition de vie. Arnett, avec sa théorie de l'âge adulte (2004), et Marcotte, Cloutier et Fortin (2010), dans l'appropriation qu'ils font de celle-ci dans le contexte de la FGA, apportent un éclairage sur cette période importante de l'adulte en quête de son insertion sociale. Ce concept, bien défini par les auteurs, nous permet de statuer que l'adulte engagé dans ce processus démontre résilience et persévérance à long terme et remet en question la notion d'un parcours qui se caractériserait par des phases successives de décrochage et de raccrochage.

Nous appuyant sur notre savoir expérientiel issu de 20 ans de pratique auprès de ces adultes, il s'est agi d'objectiver alors le savoir expérientiel des intervenants pédagogues et de leurs apprenants et, ce faisant, d'établir des questionnaires d'enquête dont l'objet était de répondre aux questions de recherche. Cette orientation prend appui sur la notion de praxis qui est l'un des cinq savoirs que Van der Maren (1996) distingue en sciences de l'éducation et dont le pôle est du domaine du savoir pratique plutôt que du domaine du savoir théorique, mais qui conduit au savoir stratégique par lequel se comprend le lien qu'il induit entre les deux types de savoirs. Comme les apprenants adultes et les intervenants (ici une enseignante et une orthopédagogue) sont en mesure de décrire également leur expérience, il nous est apparu intéressant de les faire contribuer à la construction des savoirs. 


\section{Objectifs général et spécifiques}

Ce projet comprend un objectif général et trois objectifs spécifiques en lien avec les questions de recherche.

\section{Objectif général 1 (0G-1) : Établir le portrait du parcours scolaire et de vie d'adultes (portrait ethnopédagogi- que) qui terminent leur 5 e année du secondaire à la FGA.}

\subsection{Objectif spécifique 1 (OS-1)}

Définir les liens entre persévérance scolaire et insertion sociale en identifiant les caractéristiques, les éléments facilitateurs et les obstacles des parcours scolaires et de vie sur le plan psychologique, pédagogique et social des apprenants.

\subsection{Objectif spécifique 2 (OS-2)}

Définir les liens entre pédagogie, obtention d'un diplôme d'études secondaires et insertion sociale.

\subsection{Objectif spécifique 3 (OS-3)}

Recueillir le point de vue des intervenants sur les questions suivantes : parcours d'enseignante, pédagogie et persévérance à la FGA et transition sociale.

\section{Méthodologie de recherche}

Cette recherche s'appuie sur l'ethnographie d'enquête et se sert à la fois de l'enquête écrite et de l'enquête orale, lesquelles se destinent à la documentation d'un savoir auprès de participants à la recherche nommés informateurs (Izard, 2000; Roberge, 1991). La technique du récit de vie est aussi exploitée pour comprendre les caractéristiques intrinsèques de l'adulte en poursuite d'un cheminement. Elle est un outil de collecte où le participant fait état de son expérience et a prouvé quelle est pertinente pour la production de parcours scolaires et de transition. Le récit de vie étant en soi une forme de résultats; nous en avons construit cinq. Ces récits ont aussi été utiles pour croiser les résultats, ainsi que le parcours à l'école secondaire ou à l'éducation des adultes, dont les résultats seront présentés ici. La documentation de ce savoir est rendue possible par la relation d'enquête établie entre le chercheur, nommé enquêteur, et les informateurs. La production de connaissances, issue de ce processus, repose sur des objectifs de recherche focalisés et des instruments adaptés au contexte et au profil des informateurs. En ethnographie d'enquête, une grande importance est accordée à la production du savoir comme étant un processus partagé entre informateurs et enquêteurs : il est question ici du concept de coconstruction des connaissances (Lévesque, Cloutier et Cunningham, 2009). L'ethnographie a été adoptée par plusieurs recherches récentes en éducation des adultes, parce quelle garantit la reconnaissance de l'apport des informateurs à cette construction des connaissances. Elle est pertinente pour approfondir des thématiques qui n'ont pas été traitées et peut se dérouler dans des conditions non onéreuses, notamment parce qu'elle peut se réaliser auprès d'un nombre limité d'informateurs. 


\section{Questionnaires de recherche}

Cinq questionnaires ont servi d'outils de collecte. Ces questionnaires étaient destinés à la coconstruction des connaissances avec des adultes et ont permis de répondre aux objectifs de la recherche.

Q.1 Questionnaire ethnopédagogique (apprenants) : Il comprend 100 courtes questions servant à l'établissement de 11 profils et permettant aux adultes de composer leur portrait de parcours scolaire et de vie d'apprenant. Il est conçu pour la composition d'un récit scolaire et de vie nommé récit ethnopédagogique, parce que les questions sont focalisées sur la perception pédagogique du fait scolaire. Conformément au modèle andragogique de Bélanger (2011), il s'alimente aux multiples dimensions qui composent la vie de l'adulte. Profil $1:$ données sociographiques de base; profil 2 : parcours à l'éducation des adultes; profil 3 : situation de vie; profil 4 : travail; profil 5 :TIC; profil 6 : habiletés créatives; profil 7 : parcours au secondaire; profil 8 : parcours au primaire; profil 9 : garderie et âge préscolaire; profil 10 : famille et vie scolaire; profil 11 : hygiène de vie. Questionnaire de type ethnographique oral et écrit, passé en groupe.

Q2 Rôle de la relation pédagogique à la FGA (apprenants) : Composé de 15 questions semiouvertes à répondre par écrit, ce questionnaire permet de documenter la perception qu'ont les informateurs de la relation pédagogique à la FGA relativement à l'obtention du DES. Questionnaire écrit, passé en groupe.

Q3 Questionnaire sur la persévérance à la FGA et l'insertion sociale (apprenants) : Il comprend 15 questions semi-ouvertes à répondre par écrit. Il brosse la perception quont les informateurs de leur degré d'insertion sociale à travers une approche dynamique les rapportant à leur passé, à leur présent et à leur futur, et ce, en relation avec leur parcours de persévérance à la FGA. Questionnaire écrit, passé en groupe.

Q4 Les rôles d'orthopédagogue et d'enseignante étant distincts, deux questionnaires ont été conçus. ${ }^{1}$ Questionnaires oraux individuels.

Q4.1 Le questionnaire destiné à l'enseignante de français comprend deux volets dont le premier (23 questions) porte sur son parcours d'enseignante, la pédagogie et l'enseignement individualisé et le second ( 5 questions) porte sur l'insertion sociale des apprenants.

Q4.2 Le questionnaire destiné à l'orthopédagogue comporte deux volets dont le premier (5 questions) fait état de son parcours d'enseignante et le deuxième (20 questions) permet de documenter en profondeur son intervention.

\section{Informateurs et outils de collecte de données}

Les informateurs ont été recrutés au sein d'un centre d'éducation des adultes de la région du Bas-SaintLaurent, en milieu rural. Nous avons interrogé neuf personnes : sept apprenants, une enseignante et une orthopédagogue. L'enquête a eu lieu en mars 2011 et en mai 2011. Les participants apprenants se devaient d'être des finissants sur le point d'obtenir un diplôme d'études secondaires et les intervenants, d'œuvrer auprès des apprenants adultes du centre. 


\section{Portrait ethnographique (voir Q1)}

Cinq adultes âgés de 18 à 30 ans, trois femmes et deux hommes, ont participé à cette portion de la recherche; c'est-à-dire la presque totalité des adultes finissants de ce centre. Ils nous ont été présentés par l'enseignante de français participant à l'étude.

\section{Pédagogie et insertion sociale (voir Q2 et Q3)}

Six adultes, trois femmes et trois hommes, ont participé à cette portion de la recherche, trois nouveaux membres se sont rajoutés alors que deux anciens membres se sont retirés. Ces participants nous ont également été présentés par l'enseignante de français.

\section{Parcours d'enseignante, pédagogie et persévérance à la FGA et transition sociale (voir Q4.1 et Q4.2)}

Deux intervenantes ont participé à cette portion de la recherche. Nous avons choisi d'interroger l'enseignante de français, parce qu'elle joue un rôle essentiel dans le développement des compétences linguistiques demandées sur le marché du travail et que l'enseignement du français suppose une certaine proximité affective avec l'apprenant du fait des textes remis, des confidences écrites, des débats d'idées et autres activités que commande le Programme de formation de l'école québécoise (MELS, 1998 à 2010). Nous avons également interrogé l'orthopédagogue sur son rôle au sein du centre d'éducation des adultes et sur son intervention auprès des adultes. Comme professionnelle, elle possède la posture pour faire état des enjeux psychopédagogiques et andragogiques qui touchent les adultes. Ces deux intervenantes nous ont été présentées par le directeur du centre.

\section{Méthode d'analyse des données}

Nous avons analysé les résultats selon trois axes. L'axe 1 est celui des apprenants; l'axe 2, celui de l'enseignante; l'axe 3, celui de l'orthopédagogue. Un double traitement est possible : un traitement horizontal et un traitement vertical; traitements que nous expliquons plus bas.

\section{Tableau 1}

Axes d'analyse des données recueillies

\begin{tabular}{ccc}
\hline Axe 1 & Axe 2 & Axe 3 \\
\hline Adulte(s) apprenant(s) & Enseignante de français & Orthopédagogue \\
\hline Questionnaire 1 & Questionnaire 4.1 & Questionnaire 4.2 \\
\hline Portrait ethnopédagogique & Parcours à la formation générale des adultes & Parcours à la formation générale des adultes \\
\hline $\begin{array}{c}\text { (Parcours scolaire et parcours } \\
\text { de vie) }\end{array}$ & Enseignement individualisé & Collaboration et diagnostic \\
(11 profils) & & \\
\hline
\end{tabular}




\begin{tabular}{|c|c|c|}
\hline & $\begin{array}{c}\text { Relation pédagogique et pédagogie } \\
\text { Réforme }\end{array}$ & $\begin{array}{c}\text { Relation pédagogique et pédagogie } \\
\text { Enseignement individualisé } \\
\text { Réforme }\end{array}$ \\
\hline Questionnaires 2 et 3 & Questionnaire 4.1 & Questionnaire 4.2 \\
\hline $\begin{array}{l}\text { Enseignement individualisé } \\
\text { Relation pédagogique } \\
\text { Insertion sociale }\end{array}$ & Insertion sociale & Insertion sociale \\
\hline
\end{tabular}

\section{Axe 1 de l'adulte apprenant}

L'analyse de l'axe 1 repose sur un traitement des données qui se veut tant vertical et qu'horizontal. Rappelons que le questionnaire 1, Portrait ethnopédagogique, a pour but de recueillir les éléments du parcours scolaire et du parcours de vie de l'adulte l'ayant conduit à prolonger ses études secondaires (question de recherche 1). Pour le traitement vertical, nous avons transcrit les données des dix profils; puis, nous avons additionné chacun de ces profils pour permettre la construction du récit ethnographique de vie de chaque apprenant. Pour le traitement horizontal, nous avons combiné entre elles les données des dix profils pour tous les participants : l'on peut lire et interpréter les résultats globalement ou alors isoler certains profils, ici, le parcours au secondaire et le parcours à l'éducation des adultes. Ce même modèle a été repris pour les questionnaires 2 et 3.

\section{Axe 2 et axe 3 de l'enseignante de français et de l'orthopédagogue}

En ce qui a trait aux intervenantes, les données recueillies ont été transcrites en relation avec les questions posées puis traitées dans leur globalité. Les catégories clefs ont été assignées aux éléments prévus dans les axes 2 et 3 . Par cette méthode d'analyse, nous répondons à la question de recherche 4 .

\section{Considérations éthiques}

Les données ont été anonymisées, chacun des participants ayant reçu un nom fictif. Cette recherche a reçu un certificat d'éthique émis par le Bureau de la recherche scientifique de l'Université de Montréal.

\section{Résultats de recherche}

\section{Axe 1 : Portrait ethnopédagogique des apprenants}

Un des éléments qui ressort de l'analyse de ce portrait est le caractère complexe des variables en lien avec la prolongation des études secondaires. Faute d'espace ici, nous utilisons les données qui ont trait au parcours au secondaire et à celui de l'éducation des adultes. Notons cependant que les 
récits ethnopédagogiques sont très parlants : les participants à la recherche ont rapporté dans les commentaires qu'ils étaient surpris de comprendre leur cheminement scolaire ou de vie propre.

\section{Parcours au secondaire}

Le parcours au secondaire illustre les conséquences d'un parcours sensible au primaire. Alexandra, 18 ans : quatre déménagements, redoublement; Stéphanie, 21 ans : redoublement, diagnostic de troubles d'apprentissage, ostracisme et victimisation; Mathieu, 27 ans : déménagement, aucun retard scolaire, mais troubles de comportement et agressivité; Chantal, 30 ans : plusieurs écoles, redoublement, problèmes d'intégration, de rejet et de victimisation; Maxime, 30 ans : parcours scolaire normal, mais perturbations familiales. Ce même parcours à l'école secondaire se veut également l'expression d'une période au sein de laquelle l'apprenant devenu maintenant adulte a connu des tribulations de toutes natures qui se sont répercutées dans son cheminement scolaire. Alexandra attribue ses difficultés à un déménagement lors de la $4^{\mathrm{e}}$ année du secondaire. Reclassée en $3^{\mathrm{e}}$ année du secondaire, elle abandonne ses études l'année suivante. Elle regrette non cet abandon, mais le déménagement qui a provoqué cette situation. Stéphanie a repris sa $2^{\mathrm{e}}$ et sa $4^{\mathrm{e}}$ années du secondaire, son trouble d'apprentissage l'a suivie et elle a souffert de problèmes médicaux. Son lot quotidien s'est constitué de moqueries, de menaces et de violence venant de ses camarades. Son expérience a été dégoûtante, éccurante et frustrante; trop vieille pour poursuivre, elle s'est inscrite aux adultes et affirme qu'elle aurait dû le faire avant pour s'éviter le traumatisme dont elle a souffert. Mathieu ne présente pas de retards scolaires, mais ses problèmes comportementaux se poursuivent et il rate souvent l'école. Il quitte l'école secondaire en $4^{\mathrm{e}}$ année du secondaire pour des raisons financières, encouragé par son père. Il regrette cette décision. Les problèmes de victimisation de Chantal s'arrêtent au secondaire, mais son retard se poursuit (intégration d'un programme d'apprentissage individuel et résultats scolaires faibles); c'est son conjoint manipulateur qui la conduit à arrêter l'école en $4^{\mathrm{e}}$ année du secondaire, décision qu'elle ne regrette pas, car elle s'est construite comme adulte (école de la vie). Elle ne peut pas affirmer si elle reprendrait la même décision, puisque cette décision a aussi forgé son identité. Maxime a redoublé plusieurs fois au secondaire et avoue avoir un trouble de comportement. Faute de motivation, il quitte l'école. Il regrette sa décision, car il a perdu du temps, mais en même temps ne la regrette pas parce qu'il est devenu l'homme qu'il est. Il reprendrait la même décision : la motivation et un objectif d'avenir auraient pu (l)'empêcher de quitter l'école secondaire.

\section{Éducation des adultes}

L'atteinte du diplôme secondaire (finir ce qui est commencé) et le besoin de travailler dans de meilleures conditions constituent les deux éléments relevant de la démarche d'inscription à l'éducation des adultes. Les attentes vis-à-vis des enseignants découlent de ces objectifs : aide à la compréhension, bonnes explications, enseignement efficace, variabilité des techniques d'enseignement et amélioration des compétences linguistiques. La description du milieu est favorable. Avantages organisationnels : avancement à son rythme, individualisation du travail, autonomie, disponibilité des professeurs, atmosphère propice aux études et accélération des études. Avantages andragogiques : maturité, responsabilité de ses actes et libre-choix. Avantages psychologiques : climat familial, esprit d'équipe 
et solidarité. Il est possible de croire que les centres d'éducation des adultes agissent comme lieux de passage et lieux de transition d'une étape traumatisante (le parcours) à une étape de réparation (la formation générale des adultes). Cette transition est directement en lien avec l'insertion sociale : détenir un diplôme essentiel, pouvoir entreprendre d'autres études et exercer un travail satisfaisant.

\section{Axe 1 - Pédagogie et insertion sociale du point de vue des apprenants}

Les informateurs caractérisent clairement la pédagogie qu'ils vivent. Leurs descriptions de l'enseignement individualisé et de la pédagogie de l'enseignement individualisé permettent d'affirmer qu'ils distinguent entre elles ces variables. Ils apprécient de façon positive la relation pédagogique qui a cours, le climat d'études et leur centre. On constate une évolution de la notion d'insertion sociale. Les connaissances objectives qu'ils acquièrent en français et en mathématiques sont considérées par eux comme importantes : ils ont un sentiment d'autodirection qui leur est associé et qui encourage leur persévérance à terminer des études et à considérer comme davantage possible une insertion sociale égalitaire, donc une transition de vie ou de formation.

\section{Axe 2 - Enseignante de français : parcours à la formation générale des adultes, pédagogie et insertion sociale}

Comme plusieurs pédagogues du milieu, l'enseignante de français est arrivée à cet exercice selon une atypie du parcours. En d'autres termes, possédant un brevet d'enseignement au primaire, elle s'est retrouvée par hasard dans un centre d'éducation des adultes. La formation à cette réalité s'est donc effectuée sur le tas et par la formation continue de la commission scolaire. Elle occupe un poste à statut précaire, comme c'est souvent le cas à la formation générale des adultes. L'enseignante distingue très bien entre pédagogie de l'enseignement individualisé et enseignement individualisé. Elle établit quels sont les paramètres qui caractérisent sa relation pédagogique avec les adultes de sa classe et le type de pédagogie qu'elle préconise. Il s'agit d'une pédagogie ouverte en même temps que centrée sur le développement scolaire et global de chacun de ses adultes. En connaissant les caractéristiques d'apprentissage de ceux-ci, elle favorise la persévérance de chacun dans son parcours de transition. Elle possède également un jugement global sur la réforme de la formation générale des adultes et sur les nouveaux manuels didactiques et examens que celle-ci implique. Considérant que l'enseignement individualisé doit être vu comme une pédagogie de la différenciation pédagogique, elle s'inquiète d'une réforme qui cadrerait mal avec la composition multiniveaux de sa classe et avec l'utilisation sociocognitiviste qu'elle fait de l'enseignement individualisé. Enfin, sa vision contrastée de l'insertion sociale de chacun des participants permet de juger du caractère réaliste ou non de la voie choisie par chacun.

\section{Axe 3 - Orthopédagogue: parcours à la formation générale des adultes, thématique générale, enseignement indivi- dualisé, relation pédagogique, réforme et insertion sociale}

On constate la même atypie du parcours pour l'orthopédagogue, le hasard l'ayant également conduite à postuler à la formation générale des adultes. L'analyse de l'entrevue permet de mieux saisir le rôle des intervenants : orthopédagogue, enseignants, travailleuse sociale ainsi que celui des services d'entrée 
en formation: de la passerelle avec le secondaire au régulier à l'intégration des nouveaux arrivants à la francisation. Elle porte un jugement modulé sur l'enseignement individualisé, le déclarant moins performant pour les adultes présentant de graves difficultés scolaires et le déclarant plus performant dans un contexte où il s'agit de finissants. Elle possède un discours andragogique poussé en ce qui regarde la caractérisation des troubles et des difficultés d'adultes inscrits dans le milieu : incidences de la dyslexie chez l'adulte, troubles de comportement, fragilité de la santé mentale, consommation de drogues, atteinte aux facultés et limitations cognitives. Un autre élément intéressant est le portrait qu'elle construit de la réforme et des dérives de certains outils didactiques (comme en mathématiques de $1^{\text {re }}$ et de $2^{\mathrm{e}}$ années du secondaire), considérant que la nouvelle approche conduit à l'échec pour plusieurs.

\section{Discussion des résultats}

Nos résultats rejoignent les résultats obtenus par d'autres chercheurs actifs dans notre domaine. La méthodologie de type ethnographique conduit à une recension des données complexes et abondantes. Ces données portent autant sur les adultes que sur leurs intervenantes. Le portrait ethnopédagogique des adultes permet de valider l'idée que plusieurs facteurs provenant du parcours scolaire ou du parcours de vie peuvent affecter le cheminement des études ainsi que l'insertion sociale : Marcotte et Ringuette (2011) l'ont souligné ainsi que Rousseau et al. (2010) ou Potvin et Leclercq (2010). À la différence cependant des résultats de ces chercheures, le portrait ethnographique des adultes interrogés est organisé de surcroît en récit de vie, ce qui permet une vision microsystémique - et je dirais humanisée - de leur situation. Le fait d'aborder la question de l'insertion sociale permet de considérer l'évolution de la perception des adultes en relation avec l'atteinte de la fin des études secondaires; une donnée que Potvin et Leclercq (2010) peuvent, par exemple, difficilement mesurer de la même manière avec des apprenants très fragiles et possédant une littératie précaire. En ce qui a trait à la pédagogie en cours à la formation générale des adultes, nos résultats concordent avec ceux recueillis par Marcotte et al. (2010) et Rousseau et al. (2010) : appréciation positive des enseignants, souscription à l'enseignement individualisé et attachement aux services reçus dans les centres d'éducation des adultes. Cependant, l'orthopédagogue rejoint les préoccupations de Potvin et Leclerq (2010) en ce qui a trait aux adultes demandant une intervention orthopédagogique de groupe (enseignement magistral). Par contre, aucune des études n'a abordé de façon très approfondie les pratiques pédagogiques : rôle de la pédagogie, rôle de l'enseignant, rôle de l'orthopédagogue, définition de la pédagogie de l'enseignement individualisé. Ces conclusions ouvrent la voie à des recherches qui pourraient porter sur l'étude du profil réel des apprenants et sur les parcours ethnopédagogiques de ceux-ci. D'un point de vue épistémologique, nous désirerions en outre faire état avec davantage d'acuité de méthodologies de recherche utiles à l'andragogie et destinées à la construction d'un savoir scientifique qui puise aux sources de l'ethnoscience et qui reconnaît ainsi l'apport de l'adulte, même faiblement scolarisé, au savoir sociétal en général. 


\section{Conclusion}

Notre article a fait état des résultats d'une recherche ethnographique ayant été conduite en milieu rural auprès d'adultes en fin de formation et auprès de leurs intervenantes. Pour les apprenants, le but était d'établir le portrait ethnopédagogique d'adultes finissants d'une même classe, de sonder leur perception au sujet des pratiques pédagogiques en cours dans leur milieu et de comprendre la perception qu'ils ont de leur insertion sociale. On ciblait ici autant la compréhension des éléments de la vie des apprenants qui peuvent expliquer la prolongation de leur parcours scolaire au secondaire que les causes de leur persévérance en lien avec la relation pédagogique, la pédagogie de l'enseignement individualisé et le désir manifeste d'insertion et de transition sociale. Pour les intervenants, le but ciblait la mise à jour des pratiques et du vécu pédagogiques : description de la relation pédagogique, définition de la pédagogie de l'enseignement individualisé (comme différenciation) et perception de la capacité d'insertion sociale des apprenants. Une prochaine étape se déroulera au sein de l'école montréalaise. Les outils d'enquête, se destinant à des adultes en formation et s'appuyant sur les données du milieu, seront présentés dans le cadre d'une approche ethnographique en sciences de l'éducation et impliqueront que l'on suive ces apprenants dans leur transition au travail ou aux études collégiales.

\section{Références}

Arnett, J. J. (2004). Emerging adulthood: The winding road from the late teens through the twenties. New York, NY : Oxford University Press.

Bélanger, P. (2011). Theories in adult learning and education. Farmington Hills, MI : Barbara Budrich Publishers.

Bélanger, P. et Voyer, B. (2004). L'aide à l'expression de la demande éducative en formation générale et l'accueil de cette demande dans les commissions scolaires du Québec. Repéré à http://www.treaqfp.qc.ca/103/PDF/Expression.pdf

Bélanger, P., Carignan-Marcotte, P. et Staiculescu, R. (2007). La diversité des trajectoires et la réussite éducative des adultes en formation de base. Repéré à https://parcours.uqam.ca/upload/files/Observatoire/SystemeEducatif/obs belanger reussiteeducative.pdf

Carré, P. et Moisan, A. (dir.) (2002). La formation autodirigée : aspects psychologiques et pédagogiques. Paris : L'Harmattan.

Comité d'étude sur l'éducation des adultes. (1964). Rapport du Comité d'étude sur l'éducation des adultes (Rapport Ryan). Québec, QC : Gouvernement du Québec.

Commission d'étude sur la formation des adultes (CÉFA). (1982). Apprendre : une activité volontaire responsable. Énoncé d'une politique globale de l'éducation des adultes dans une perspective d'éducation permanente. Québec, QC : CÉFA.

Commission des États généraux sur l'éducation. (1996). Les États généraux sur l'éducation, 1995-1996. Rénover notre système d'éducation : dix chantiers prioritaires. Québec, QC : Commission des États généraux sur l'éducation.

Commission royale d'enquête sur l'enseignement dans la province de Québec. (1963). Rapport de la Commission royale d'enquête sur l'enseignement dans la province de Québec (Rapport Parent). Québec, QC : Ronlads Federated Ltd.

CONFINTEA VI. (2009, décembre). Vivre et apprendre pour un futur viable - l'importance de l'éducation des adultes. Communication présentée à la Sixième conférence sur l'éducation des adultes, Belém, Brésil. Repéré à http://www.unesco.org/archives/multimedia/?s=films details\&pg=33\&vo=3\&vl=Fre\&id=2686

Conseil supérieur de l'éducation. (1994). Un régime pour l'éducation des adultes dans les commissions scolaires. Avis au ministre de l'Éducation. Repéré à https://www.cse.gouv.qc.ca/fichiers/documents/publications/Avis/50-0393.pdf

Conseil supérieur de l'éducation, Table des responsables de l'éducation des adultes et de la formation professionnelle des commissions scolaires du Québec (TREAQFP) et Ministère de l'Éducation. (2000). La formation de base en éducation des adultes (L'exemple de l'Orégon). 
Doray, P. et Bélanger, P. (2005). Société de la connaissance, éducation et formation des adultes. Éducation et sociétés, 2005/1(15), 119-135. http://dx.doi.org/10.3917/es.015.0119

Doucet, M., Gauthier, I., Bizot, D. et Maltais, D. (2013). L’identité professionnelle des enseignants à la formation générale des adultes : trajectoires, défis et enjeux éducationnels. Communication présentée au Symposium " Parcours et trajectoire à l'éducation des adultes, regards croisés » du Congrès international de l'AECSE, Montpellier, France.

Gouvernement du Québec. (2000). Loi sur l'instruction publique (L.R.Q. chapitre I-13.3). Québec, QC : Bibliothèque nationale du Québec.

Gouvernement du Québec. (2002). Apprendre tout au long de la vie. Politique gouvernementale d'éducation des adultes et de formation continue. Repéré à http://www.mess.gouv.qc.ca/publications/pdf/SR politique gouv education adultes.pdf

Inchauspé, P. (1999). Vers une politique de formation continue. Québec, QC : Ministère de l'Éducation.

Inchauspé, P. (2000). Pour une politique de l'éducation des adultes dans une perspective de formation continue. Repéré à http://collections.banq.qc.ca/ark:/52327/bs42073

Izard, B. (2000). Méthode ethnographique. Dans Dictionnaire de l'ethnologie et de l'anthropologie (p. 470-475). Paris : Presses universitaires de France.

Lévesque, C., Cloutier, É. et Cunningham, J. (2009). Working towards the coproduction of knowledge: a research partnership involving Aboriginal people. Barcelone : Global University Network for Innovation et UNESCO.

Long, H. B. (1989). Self-directed learning: emerging theory E practice. Norman, OK : Oklahoma Research Center for Continuing Professional and Higher Education.

Marcotte, J., Cloutier, R. et Fortin, L. (2010). Portrait personnel, familial et scolaire des jeunes adultes émergents (16-24 ans) accédant aux secteurs adultes du secondaire : identification des facteurs associés à la persévérance et à l'abandon au sein de ces milieux scolaires. Repéré à http://www.frqsc.gouv.qc.ca/documents/11326/535055/PT MarcotteI rapport+2010 PRS+16-24+ans-secteur+adulte+secondaire/5444d2ff-1ee2-4cea-9bd0-9e10d6fe 7713

Marcotte, J. et Ringuette, D. (2011). Étude exploratoire des liens entre l'identité et l'implication scolaire des jeunes de 18 à 24 ans en formation générale aux adultes. Revue de psychoéducation, 40(2), 241-260.

Marcotte, J., Villatte, A. et Lévesque, G. (2014). La diversité et la complexité des jeunes (16-24 ans) inscrits à l'éducation des adultes au Québec : enquête et essai de typologie. Revue des sciences de l'éducation, 40(2), 253-285. http://dx.doi.org/10.7202/1028421ar

Ministère de l'Éducation, du Loisir et du Sport (MELS). (2008). Le renouveau pédagogique en formation générale des adultes. Québec, QC : Gouvernement du Québec.

Ministère de l'Éducation, du Loisir et du Sport (MELS). (2009-2011). Statistiques de l'éducation. Québec, QC : Gouvernement du Québec.

Ministère de l'Éducation, du Loisir et du Sport (MELS) et Direction de l'éducation des adultes et de l'action communautaire (DEAAC). (2007). Programme de la formation de base commune. Québec, QC : Gouvernement du Québec.

Potvin, M., Voyer, B. et Bourdon, S. (2014). Les transformations et défis actuels de la formation générale des adultes (Numéro thématique). Revue des sciences de l'éducation, 40(2).

Roberge, M. (1991). Guide d'enquête orale. Québec, QC : Gouvernement du Québec.

Rogers, C. R. (1973). Liberté pour apprendre? Paris : Dunod.

Rousseau, N., Théberge, N., Bergevin, S., Tétreault, K., Samson, G., Dumont, M., et Myre-Bisaillon, J. (2010). Léducation des adultes chez les 16 à 18 ans : La volonté de réussir l'école... et la vie! Éducation et francophonie, 38(1), 154-177. http://dx.doi.org/10.7202/039985ar

Royer, É. (2014). Le décrochage scolaire, prévisible? Repéré à http://www.ameqenligne.com/news_detail.asp?ID=525020 
Solar, C. et Thériault, M. (2013). Adult education and training in francophone Canada. Dans T. Nesbit, S. M. Brigham, N. Taber et T. Gibb (dir.), Building on critical traditions: Adult education and learning in Canada. Toronto, ON : Thompson Educational Publishing.

Table des responsables de l'éducation des adultes et de la formation professionnelle des commissions scolaires du Québec (TRÉAQFP). (2013). Synthèse du plan d'action 2013-2014 de la TRÉAQFP. Repéré à http://treaqfp.qc.ca/plan action/Synthese plan action 13 14 5nov13.pdf

Villemagne, C. et Myre-Bisaillon, J. (dir.) (2015). Les jeunes adultes faiblement scolarisés. Québec, QC : Presses de 1'Université du Québec.

\section{Note de bas de page}

1 Les interviews auprès d'intervenants enseignantes ou orthopédagogues de la formation générale des adultes sont rarissimes (Bélanger, Carignan-Marcotte et Staiculescu, 2007; Voyer, 2002). Il nous apparaît que ce manque doit être comblé, aussi nos questionnaires ont-ils été conçus pour recueillir des données servant à documenter le savoir expérientiel et stratégique de ces intervenantes.

\section{Pour citer cet article}

Thériault, M. (A.) (2016). Apprendre et enseigner à la formation générale des adultes : une recherche ethnopédagogique auprès de finissants et finissantes ainsi que d'enseignantes [Learning and teaching at Adult Education: ethnopedagogical research with close graduate and teachers]. Formation et profession, 24(3), 32-45.

http://dx.doi.org/10.18162/fp.2016.381 